will be refracted on entering the ionised portions and, upon reaching a height at which the density of ionisation is sufficiently great, the deviation will be such as to return the waves to the earth's surface. It has been found that on a wave-length of about 50 metres and using an antenna power of 5 kilowatts, these waves returning from the ionosphere reach the earth and affect a receiver at a minimum distance of the order of 2,500 miles. Since under the same conditions the ground wave will only be detectable at distances up to about 90 miles, there will be an annular space around the emitting station having inner and outer radii of 90 miles and 2,500 miles respectively in which no signals will be detectable. This is commonly known in radio engineering circles as the 'skip' effect and the larger radius above is termed the 'skipped distance'. As the wave-length is increased, this distance decreases steadily owing to the greater refraction effects taking place in the upper atmosphere; at the same time the range of the ground wave increases, and finally the two overlap for wave-lengths approximately in the shorter broadcasting band of 200 metres to 500 metres. Under these conditions, both ground and atmospheric waves reach the receiving station, and it is the interference effects resulting from these two sets of waves which cause the well-known phenomena of fading in broadcast reception, and of the variation of bearing in radio direction-finding.

In the later portion of his lecture, Sir Frank Smith described how the radio investigator has recently directed his attention to obtaining more conclusive proofs of the existence of the ionosphere and to the quantitative measurement of its properties; that is, to obtain information which the geophysicist has been unable to supply. Methods employed in other branches of physics have been adapted to radio communication for this purpose. One of these makes use of the interference effects obtained between the ground and atmospheric waves to determine the height of the ionosphere. The same objective has also been attained by using an echo method to measure the time taken by a pulse of electric waves to travel up to the ionised deflecting layer and return to earth. Owing to the very high velocity of the waves, this time interval is short-about one-thousandth of a second-and the cathode ray oscillograph has proved an invaluable tool in the determination of this quantity.

Working in this manner, the radiotelegraphist has conclusively proved the existence of the Kennelly-Heaviside layer in the ionosphere at an average height of about $100 \mathrm{~km}$. The measurements showed that for most nights the height of this ionised layer gradually increases, a maximum value being reached about one hour before sunrise. Further investigation showed that on certain occasions and with wave-lengths less than 400 metres, the height of the deflecting layer suddenly changes from $100 \mathrm{~km}$. to about $230 \mathrm{~km}$. It then became evident that on such occasions the density of ionisation in the Kennelly-Heaviside layer is insufficient for the deflection of the shorter waves, which thus penetrated this layer, but are ultimately deflected by a second layer above the first, the existence of which had not previously been suspected. This upper layer has been named after Prof. E. V. Appleton, who, with the co-operation of the Radio Research Board, has played the leading part in Great Britain in the investigations just described.

Having fixed the heights of these two important regions of the atmosphere, it has next been found possible to determine the density of the ionisation in these regions by ascertaining the limiting wavelength or frequency of the waves which will just penetrate the layer. Experiments carried out in this manner have shown that the ionisation is at its maximum about noon and decreases steadily as sunset is approached. After sunset, a rapid fall in ionisation takes place until, at about two hours afterwards, its value is only about onetenth of the maximum, and this then remains reasonably constant until shortly before sunrise. Two further facts which emerge from the work are that there is very little ionisation between the Kennelly-Heaviside and the Appleton layers, and that, for wave-lengths less than about 8 metres, the waves penetrate both layers and there is as yet no evidence of their return to earth from the upper atmosphere. As to the cause of the ionisation, some data obtained during the solar eclipse of August 31, 1932 appear to establish the fact that the principal ionising agent for the two layers is ultra-violet light. The possible bombardment of the earth by neutral particles is not yet wholly excluded, but undoubtedly the evidence so far is against it.

The review provided in this lecture shows very clearly that the modern radio research worker is obtaining detailed quantitative knowledge of certain portions of the atmosphere in a manner which was beyond the reach of the former geophysical methods of attack.

\title{
Hydrogenated Motor Lubricants
}

$\mathrm{T}$ HE recent display of advertisements has directed public attention to a new oil, "Essolube", a product representing the muchdiscussed process of hydrogenation successfully applied to the refining of petroleum. This particular lubricating oil is the product of many years research, involving much expenditure and the laying down of large-scale plant. The entry of hydrogenation as an established commercial process in the oil industry is an event of first-class technical and economic importance, and in many respects it will be a surprise to most people that the first satisfactory synthetic product in this field is a lubricant, a class of material distinguished for 
its extreme variability and one of the most sensitive (in performance) of the whole range of petroleum distillates.

Broadly speaking, it is demanded that a highquality lubricating oil should possess a good viscosity index, a low pour point, a wide range of stability, high flash point and low earbon value; these properties are claimed as entirely characteristic of the synthetic product. A good viscosity implies an oil which changes least in characteristics with temperature range, making for easy starting of an engine in cold weather, reducing wear of the moving parts and, at the same time, ensuring that under operating conditions an adequate oil film is preserved round the piston; these factors naturally affect the question of oil consumption. The essence of stability is the ability of the oil to preserve its fundamental characteristics in performance, and the lack of tendency to form sludge or to coagulate in any manner which might tend to choke the lubricating system. The low carbon value is sought for many reasons ; chiefly to take care that, whatever carbon is formed, is of a soft and noncrystalline character, as hard carbon particles returned to the oil sump will inevitably act as an abrasive and do damage to the vital parts of the engine.

Production of modern lubricating oils is, quite apart from this new process, a highly specialised subject, concerning which much still remains to be, and is being, done in the matter of fundamental research. Possibilities of hydrogenation as applied to petroleum products have of course been long foreseen by technologists both in Great
Britain and in America, but the advent of a marketable product at this juncture is certainly a most commendable departure.

An account of this enterprising action of the Anglo-American Oil Co., the concern responsible for the introduction of this new lubricating oil, appears in the Times of April 29. Concurrently with the appearance of this product, the same Company is responsible for introducing a new method of delivery of the oil to motor engines. Supplies will be fed directly to the engine from sealed glass bottles and the displacing of the familiar tin will be an advantage in many ways since, apart from guaranteed measure, the contents can be seen and, incidentally, the shape of the bottle is such that it can be easily inverted into the oil filler of the engine, thus obviating loss by spilling, especially where such fillers are placed in awkward positions.

It is of interest to note the Company's exhibition of hydrogenation at Charing Cross Underground Station, a place, incidentally, one now instinctively associates with modern commercial enterprise in educating the public to at least some visual knowledge of commodities in everyday use which, in a vast majority of cases, are taken very much for granted. So much has been heard of, and written about, hydrogenation in the last few years without, to the lay mind, as yet any tangible expression, that the coincidence of a new synthetic hydrocarbon and the display of technique and issues involved, will do much to awaken interest in the untiring service that science renders to the community to-day.

\section{Lessons in Visualisation from the Royal Academy}

\section{By Dr. Vaughan Cornish}

CCENERY is defined as the appearance of a $\checkmark$ place from the picturesque point of view, and so, without pausing to consider what constitutes picturesqueness, we may note at once that scenery is an appearance. The science of scenery is, therefore, a science of appearances, and consequently the question whether a landscape painting is true to the facts can only be answered if we know how the physical surroundings are pictured through the agency of the eye.

There are three very different modes of picturing landscape; by the camera, by the representational school of painting, and by the imaginative school. Leaving the discussion of the last category for another occasion, let us now compare the rendering of the facts of scenery by the camera and by the school of painting hitherto dominant at the Royal Academy.

Even within the limits of monochrome, to which the camera is usually restricted, a clear photograph of a well-lit landscape is radically different from the natural picture. Not only the eye of the artist but also that of the ordinary man is selective. The natural picture of the landscape is not the sum of those features which we can see when we examine bit by bit the whole field of outlook, but a composition formed of that which we notice spontaneously. It may even be that in scenes which stir the emotions deeply, the proportion that remains unnoticed, that is to say mentally invisible, is greatest.

Largely on account of this selective action of the eye, and not merely on account of the vagueness of the image near the edge of the retina, the skilful composition of the landscape painter is in some important respects nearer than the photograph to the facts of scenery. It may be suggested in passing that the customary preference of the landscape artist for painting largely from memory, is not only on account of the troublesome variation of light in the actual scene, but also because memory is selective, and its partialities help to obliterate the inessential from the mental picture.

As an example of truth to Nature (in the wider sense) as exemplified in the school of landscape painting usually dominant at the Royal Academy, I may cite "The Shores of Clyde" (340) by 